\title{
Inter-Relações entre os Ciclos de Transmissão do Trypanosoma cruzi no Município de Bambuí, Minas Gerais, Brasil
}

\author{
Relationships Between Trypanosoma cruzi Transmission Cycles in the \\ County of Bambuí, Minas Gerais, Brazil
}

Alexandre José Fernandes '; Liléia Diotaiuti 2; João Carlos P. Dias ${ }^{1 ;}$ Álvaro José Romanha ${ }^{1} \&$ Egler Chiari ${ }^{2}$

FERNANDES, A. F.; DIOTAIUTI, L.; DIAS, F. C. P.; ROMANHA, A. F. E CHIARI, E. Relationships Between Trypanosoma cruzi Transmission Cycles in the County of Bambui, Minas Gerais, Brazil. Cad. Saúde Públ., Rio de Faneiro, 10 (4): 473-480, Oct/Dec, 1994.

This study examines recent relationships between domestic and sylvatic transmission cycles of $\underline{T}$. cruzi in the county of Bambui, MG, Brazil. In the late 1930s, Panstrongylus megistus was found in $75 \%$ of houses. Subsequently, Triatoma infestans became the predominant species, found in $20 \%$ of urban households and in more than $60 \%$ of periurban homes. With intense insecticide control campaigns between 1956 and 1969, T. infestans was eradicated from the county, transmission of Chagas' disease to man was interrupted, and P. mesgistus appeared in rural residences. Samples of $\underline{T}$. cruzi isolated by xenodiagnosis and hemoculture from 43 opossums (Didelphis albiventris) captured in both peridomiciliary and sylvatic areas were characterized by isoenzyme analysis and - regardless of isolation method - were found to present the Z1 zymodeme profile. Through the "Chagas' Disease Epidemiological Surveillance Program", from August 1986 to December 1988, 154 specimens of P. megistus were captured by the local population in both peridomiciliary and intradomiciliary environments, of which $9.8 \%$ were infected with $\underline{T}$. cruzi. In isoenzyme analyses of $l 3 \underline{T}$. cruzi strains isolated from these triatomines, six were found to be of the Z1 zymodeme (sylvatic transmission cycles) and seven were found to be of the $Z 2$ zymodeme (domestic transmission cycle). The capture of $\underline{P}$. megistus specimens in intradomiciliary environments that were naturally infected with parasites of both cycle types indicates an overlap of transmission cycles of Chagas' disease in the county of Bambui. Further evidence for the interrelationship of the two cycles was provided by the isolation of $\underline{T}$. cruzi of the $Z 2$ zymodeme from a cat and the participation of the dog as a reservoir of $Z 1$ T. cruzi. The presence of $\underline{P}$. megistus in the peridomiciliary environment represents an important link between the sylvatic and intradomestic environments serving as a carrier of $Z 1 \underline{T}$. cruzi and maintaining the transmission cycles of $Z 2 \underline{T}$. cruzi in the peridomestic and intradomestic environments thus providing the potential for a gradual reinfestation of the county if the "Epidemiological Surveillance" is interrupted.

Key words: Chagas' Disease; Trypanosoma cruzi Infection; Transmission Cycles; Reservoirs; Zymodemes

${ }^{1}$ Centro de Pesquisas René Rachou da Fundação Oswaldo Cruz. Av. Augusto de Lima, 1715, Caixa Postal 1743, Belo Horizonte, MG, 30190-020, Brasil.

${ }^{2}$ Instituto de Ciências Biológicas da Universidade Federal de Minas Gerais. Avenida Antônio Carlos, 6627, Caixa Postal 486, Belo Horizonte, MG, 31270-901, Brasil.

\section{INTRODUÇÃO}

A infecção chagásica, inicialmente uma enzootia silvestre, transmitida na natureza entre animais e triatomíneos, transformou-se em uma antropozoonose com a intrusão do homem no 
ambiente natural no qual circulava o Trypanosoma cruzi. A ocupação predatória desses espaços pelo homem, fez com que algumas espécies de triatomíneos fossem introduzidas, ativa ou passivamente, em suas habitações e se estabelecessem novos ciclos de transmissão. Dessa maneira, o homem e os animais doméseticos passaram a fazer parte da cadeia epidemiológica da doença de Chagas, com possibilidade de intercâmbio do T. cruzi entre os ciclos silvestre e doméstico (Barretto, 1979; Forattini, 1980).

A susceptibilidade do homem e dos animais domésticos ao $T$. cruzi e a proliferação de triatomíneos nas habitações propiciaram a disseminação do parasita, passando o ciclo doméstico a ter importância fundamental na expansão da infecção chagásica (Barretto, 1967), podendo existir, até mesmo, independente do ciclo silvestre. No Brasil, a endemia chagásica afeta cerca de 5 milhões de pessoas, $20 \%$ das quais apresentam cardiopatia crônica, e $10 \%$, manifestações digestivas, permanecendo a grande maioria na fase indeterminada (Dias, 1987; OMS, 1991).

Em Bambuí, oeste de Minas Gerais, os estudos da doença de Chagas iniciaram-se com a descoberta do foco por Martins et al. (1939/ 1940). Segundo Dias (1982), a doença de Chagas no município pode ser descrita em três períodos. O período inicial (1940-1956), caracterizado pelos altos índices de triatomíneos com infecção natural pelo T. cruzi, presença maciça de Triatoma infestans e elevados índices de transmissão ao homem. $\mathrm{O}$ período intermediário (1957-1970) inicia-se com os trabalhos de desinsetização, com redução do T. infestans e da transmissão da doença ao homem, apresentando momentos de reinfestação domiciliar em algumas áreas rurais. Em 1971, consolida-se a profilaxia antivetorial, e, em 1974, há a implantação da Vigilância Epidemiológica, que caracteriza o terceiro período. Nele ocorre a erradicação do $T$. infestans e a interrupção da transmissão da doença de Chagas ao homem, com o surgimento esporádico de adultos do Panstrongylus megistus nas casas rurais.

Miles et al. (1977), estudando o perfil de isoenzimas de amostras de T. cruzi de São Felipe, Bahia, Brasil, caracterizaram pela primeira vez, intrinsecamente, duas formas do parasita. O primeiro grupo, denominado zimodema $\mathrm{Z1}$, foi isolado de gambás e o vetor Triatoma tibiamaculata procedentes do ambiente silvestre; o segundo grupo, denominado zimodema $\mathrm{Z2}$, foi encontrado em pacientes chagásicos e animais domiciliados em casas infestadas pelo vetor doméstico $P$. megistus. A distinção dos dois grupos de cepas circulando independentemente sugeriu que os ciclos de transmissão silvestre e doméstico do T. cruzi em São Felipe não se superpunham. Posteriormente, Miles et al. (1978), analisando o perfil isoenzimático de amostras de T. cruzi de pacientes chagásicos de Belém, Pará, Brasil, encontraram um terceiro grupo de parasitas, denominado zimodema Z3. Foi verificada, então, pela primeira vez, por meio dos estudos isoenzimáticos, a heterogeneidade do T. cruzi infectando o homem.

Romanha (1982), com o objetivo de caracterizar cepas do T. cruzi por métodos bioquímicos, estudou 67 amostras isoladas de pacientes chagásicos crônicos e de um reservatório silvestre (Didelphis albiventris) da região de Bambuí. Por meio do perfil eletroforético de isoenzimas, foram descritas quatro populações de T. cruzi, denominadas zimodemas A, B, C e D. As correlações entre os três zimodemas descritos por Miles et al. (1977, 1978) com os de Romanha (1982) foram: a) Z1 foi semelhante aos perfis de T. cruzi isolado do gambá; b) $Z 2=Z A$; e c) $Z 3 \simeq Z C$. Não foram encontradas correlações entre os zimodemas B e D.

O perfil de isoenzimas, a análise do DNA cinetoplástico (K-DNA) por endonucleases de restrição, DNA nuclear e os anticorpos monoclonais têm sido usados para a caracterização do parasita e melhor entendimento da epidemiologia da doença de Chagas (Miles, 1983; Gibson \& Miles, 1985; Hide \& Tait, 1991; Macêdo et al. 1992). Os estudos enzimáticos têm sido utilizados para responder a algumas questões referentes à heterogeneidade populacional do T. cruzi, inter-relações dos ciclos de transmissão doméstico e silvestre e na taxonomia numérica.

Este estudo teve como objetivo definir o possível relacionamento entre os ciclos de transmissão silvestre e doméstico do $T$. cruzi em Bambuí, complementando a vasta infor- 
mação já existente sobre a epidemiologia clínica na região (Laranja, 1949; Pellegrino, 1953; Dias, 1982). Para isso foram correlacionados os perfis isoenzimáticos de amostras de $T$. cruzi isoladas de pacientes chagásicos crônicos já descritos por Romanha (1982) com aqueles de cepas isoladas de gambás (D. albiventris), triatomíneos (P. megistus) e reservatórios domésticos naturalmente infectados do município.

As amostras de T. cruzi foram isoladas por hemocultura em meio LIT (Camargo, 1964) e/ou xenodiagnóstico, de acordo com Bronfen et al. (1989), e a caracterização isoenzimática, segundo Romanha (1982).

\section{COMENTÁRIOS}

O termo zimodema (população de parasita com uma única combinação de perfis de enzimas), definido pela World Health Organization (WHO) em 1978, tem sido usado com diferentes significados. Ready \& Miles (1980) agruparam as populações de T. cruzi em zimodemas principais e admitem variações numa ou outra enzima sem que mude o zimodema; Gibson et al. (1980) consideraram amostras de T. cruzi, diferindo em uma única enzima, pertencentes a diferentes zimodemas; Tibayrenc et al. (1984) utilizaram o termo "cepa isoenzimática" como sinônimo de zimodema, mas sem levar em consideração sua importância taxonômica ou correlações com as formas clínicas da doença de Chagas. Para esses autores, uma terminologia mais precisa só será possível após o conhecimento da variabilidade isoenzimática do T. cruzi.

Os perfis isoenzimáticos de amostras de $T$. cruzi isoladas de 43 gambás de Bambuí mostraram uma população homogênea do parasita pertencente ao zimodema $\mathrm{Z} 1$, circulando entre esses animais, tanto no ambiente silvestre como no peridoméstico (Fernandes et al., 1991). As características da infecção pelo T. cruzi em gambás sugerem uma relação parasito-hospedeiro bastante equilibrada. Esse fato foi relacionado a um processo lento e gradativo de evolução que incluiria o parasitismo das glândulas anais (Deane et al., 1984; Fernandes et al.,
1989), uma vez que no lúmem dessas glândulas os parasitas estariam protegidos do sistema imune do hospedeiro (Deane \& Jansen, 1988). Deane \& Jansen (1988) sugeriram que as glândulas anais poderiam ter sido um ponto de passagem de tripanosomatídeos monogenéticos para digenéticos. Dentro dessa hipótese, poderíamos admitir que populações de T. cruzi procedentes do ciclo enzoótico, provavelmente aquelas circulando entre os gambás, poderiam ser considerados populações parentais, dando origem ao que Tibayrenc \& Ayala (1988) chamaram de clones naturais com uma estrutura populacional complexa e heterogênea.

Em Bambuí, a presença de $P$. megistus em ecótopos silvestres é relatada unicamente por Dias (1982) em palmeiras, onde este triatomíneo é esporadicamente encontrado em números reduzidos. Esse fato é surpreendente uma vez que são freqüentemente observados focos de colonização por essa espécie em ecótopos artificiais (Dias, 1965; Fernandes et al., 1992a).

No período de agosto de 1986 a dezembro de 1988, foram enviados ao Posto Avançado de Estudos Emmanuel Dias - Fundação Oswaldo Cruz (Fiocruz), em Bambuí, por meio do programa de Vigilância Epidemiológica(Dias \& Garcia, 1978), 154 triatomíneos procedentes de diversas localidades. Todos eram P. megistus, estando $9,8 \%$ positivos para $T$. cruzi. Não houve diferença estatisticamente significativa entre o número de ninfas e adultos capturados no peridomicílio ou intradomicílio. Esses dados são de grande importância epidemiológica, pois mostram de maneira clara a tendência do $P$. megistus local de colonizar ambientes próximos ao homem. Nesse sentido, observa-se que os triatomíneos procedentes dos focos naturais exercem pressão constante no peridomicílio e no intradomicílio. Entre os 13 triatomíneos capturados no ambiente artificial, dos quais se isolou e caracterizou isoenzimaticamente o $T$. cruzi, sete $(53,8 \%)$ apresentaram parasitas com perfil isoenzimático do zimodema $\mathrm{Z} 2$, característico do ciclo domiciliar (Miles et al., 1977, 1978), sendo que quatro (30,8\%) amostras foram isoladas de ninfas. Esses dados mostram claramente que a infecção desses $P$. megistus se deu em ambiente peridomiciliar e/ou domiciliar. Amostras recentes de T. cruzi isoladas de 
uma paciente e de um gato procedentes de uma residência onde ninfas e adultos de $P$. megistus foram capturados naturalmente infectados, apresentaram o mesmo zimodema Z2, demonstrando a ocorrência do ciclo de transmissão doméstico (vetor-reservatório-homem) sem a participação do ciclo silvestre.

Pôde-se evidenciar a presença de pequenas colônias de $P$. megistus no intradomicílio e de ciclos isolados de transmissão doméstico e peridoméstico do T. cruzi. Um aspecto de importância foi a notável participação do homem como fonte alimentar dos triatomíneos, uma vez que em $40 \%$ deles foi possível identificar sangue humano nos seus conteúdos estomacais, mediante a reação de precipitina (Siqueira, 1960), o que possibilita ocorrência de transmissão vetorial. O isolamento de T. cruzi com padrão de zimodema $Z 1$, característico de parasita do ciclo silvestre (Miles et al., 1977, 1978), a partir de seis outros triatomíneos, sugere que esses insetos procediam daquele ambiente. Todos os seis eram adultos, e três não apresentavam sangue em seus tubos digestivos, sugerindo longo período de jejum. A captura de somente um exemplar de $P$. megistus por residência e adulto corrobora a hipótese de recente introdução dos triatomíneos nas mesmas.

A heterogeneidade das amostras de T. cruzi isoladas de pacientes chagásicos da mesma região (Zimodemas A, B, C e D) (Romanha, 1982) e a homogeneidade das amostras silvestre que circulam entre gambás e $P$. megistus sugerem a existência na região de um ciclo e transmissão silvestre, onde o $T$. cruzi que circula nesse ambiente é isoenzimaticamente diferente do observado no ciclo de transmissão doméstico.

A análise histórico-epidemiológica realizada em Bambuí por Dias (1982) revelou que, no final dos anos 30, o P. megistus era o triatomíneo que predominava na região. Esse inseto já exercia grande importância na transmissão do $T$. cruzi, mesmo antes da entrada do T. infestans no município, hipótese fortalecida pela alta prevalência da doença de Chagas em indivíduos com idade superior a 40 anos na década de 1940. A infestação das habitações pelo $P$. megistus a essa época deu-se a partir dos focos silvestres, e o T. cruzi com perfil de zimodema
$\mathrm{Z1}$, provavelmente, foi o responsável pela infecção humana. $\mathrm{O}$ fato de pacientes chagásicos crônicos de Bambuí terem apresentado variações nos perfis isoenzimáticos em um segundo isolamento do parasita (Romanha, 1982) sugere que, durante a evolução da infecção chagásica, fatores intrínsecos relacionados ao hospedeiro vertebrado e ao T. cruzi, num processo de adaptação, poderiam ter interferido nessas variações. Alterações dos perfis isoenzimáticos das amostras procedentes de Bambuí e estudadas por Romanha (1982) foram, também, observadas por outros autores (Bahia, 1985; Carneiro et al., 1990). Não obstante, deve-se considerar o fato de nunca ter sido isolado o $T$. cruzi $\mathrm{Z1}$ de pacientes chagásicos do município de Bambuí, apesar de grande número de amostras estudadas (Romanha, 1982).

A chegada do T. infestans na região deu-se, provavelmente, no final dos anos 30 , sendo seu processo de colonização extremamente rápido (Dias, 1982). Nesse sentido, esse vetor poderia ter introduzido diferentes populações de $T$. cruzi no ciclo de transmissão doméstico em Bambuí, uma vez que esse triatomíneo, no município, sempre foi encontrado dentro das casas (Dias, 1982). Estudos realizados na Bolívia mostraram a existência de diferentes amostras de T. cruzi semelhante ao zimodema $\mathrm{Z} 2$ (Miles et al., 1977), circulando simpatricamente e sendo transmitidas pelo T. infestans, às vezes, até no mesmo ambiente domiciliar (Tibayrenc et al., 1986). Desse modo, observa-se que o $T$. infestans, circulando em ambiente doméstico e estando comumente sujeito à disseminação passiva (Zeledón, 1974), atuaria como carreador de diferentes amostras de T. cruzi que circulam nos ciclos de transmissão doméstico do parasita.

Amostras de T. cruzi isoladas dos ciclos de transmissão silvestre e doméstico da doença de Chagas do município de Bambuí, estão sendo clonadas com o objetivo de permitir melhor entendimento das diferenças observadas. Até o momento foram clonadas três amostras pertencentes ao zimodema $\mathrm{Zl}$ e duas pertencentes ao Z2. Dez clones de cada amostra foram caracterizadas bioquimicamente. Todos eles apresentaram perfis isoenzimáticos idênticos aos das amostras parentais (Fernandes et al., 1992b). Esses dados sugerem, como proposto por Ti- 
bayrenc \& Ayala (1988), que as populações de T. cruzi apresentam uma estrutura clonal e que a heterogeneidade biológica e bioquímica do parasita, entre outros fatores, teria sido resultado da adaptação por meio da seleção natural de determinados clones ao homem no ciclo doméstico e a outros hospedeiros no ambiente silvestre.

O isolamento de amostras de T. cruzi pertencentes ao zimodema $Z 1$, circulando entre gambás e $P$. megistus em ambiente peridoméstico/doméstico e silvestre indicam uma relação gambá-triatomíneo-T. cruzi Z1 e a superposição dos ciclos de transmissão da doença de Chagas nas áreas estudadas. Outra evidência da inter- relação dos ciclos pôde ser observada no isolamento de $T$. cruzi $\mathrm{Z} 2$ de um gato e a participação do cão como reservatório de $T$. cruzi Z1 (Figura 1) (Fernandes et al., 1993). A presença do $P$. megistus no peridomicílio representa importante elo entre o ambiente silvestre e o intradomicílio, servindo como veiculador do T. cruzi Z1 e na manutenção de ciclos esporádicos de transmissão do T. cruzi Z2 no peridomicílio e domicílio. Poderá ocorrer de forma gradual a reinfestação do município, com risco real de reativação do ciclo doméstico do $T$. cruzi, caso a Vigilância Epidemiológica seja interrompida, hipótese que também existe para outros municípios brasileiros.

FIGURA 1. Inter-relações entre os Ciclos de Transmissão do T. cruzi no Município de Bambuí, Minas Gerais, de Acordo com a Distribuição dos Diferentes Zimodemas (Z)

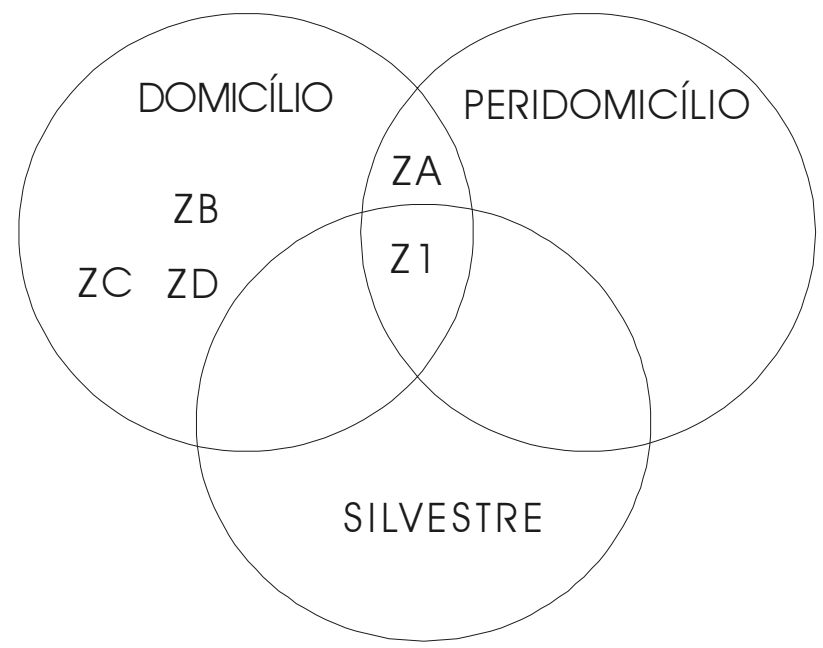

Populações de T. cruzi

Hospedeiros

ZB，ZC，ZD

$\mathrm{Z1}$

$\mathrm{ZA}$

$\mathrm{Z} 2=\mathrm{ZA}$
- Paciente chagásico crônico

- Panstrongylus mesgistus, Didelphis albiventris, cão

- Pasnstrongylus megistus, gato, paciente chagásico

- crônico 


\section{RESUMO}

FERNANDES, A. J.; DIOTAIUTI, L.; DIAS, J. C. P.; ROMANHA, A. J. \& CHIARI, E.

Inter-Relações entre os Ciclos de Transmissão do Trypanosoma cruzi no Município de Bambuí, Minas Gerais, Brasil. Cad. Saúde Públ., Rio de Janeiro, 10 (4): 473-480, out/dez, 1994.

Neste trabalho apresentamos um estudo das inter-relações recentes entre os ciclos de transmissão silvestre e doméstico do T. cruzi no município de Bambuí, Minas Gerais, Brasil. No final da década de 1930, o Panstrongylus megistus era encontrado em $75 \%$ das casas. Subseqüentemente, o Triatoma infestans tornou-se a espécie predominante, sendo encontrada em $20 \%$ das residências urbanas e em mais de $60 \%$ das periurbanas. Com as intensas campanhas de borrifação desenvolvidas entre 1956 e 1969, o T. infestans foi erradicado do município, e a transmissão da doença de Chagas ao homem, interrompida, com aparecimento de $P$. megistus em residências rurais.

Amostras de T. cruzi isoladas via xenodiagnóstico e hemocultura de 43 gambás (Didelphis albiventris) capturados em ambiente peridomiciliar e silvestre foram caracterizadas isoenzimaticamente e, independentemente da via de isolamento, apresentaram perfil de zimodema Z1. Por meio do Programa de Vigilância Epidemiológica da doença de Chagas, no período de agosto de 1986 a dezembro de 1988, 154 exemplares de $P$. megistus foram capturados pela população no peridomicílio e intradomicílio rural, estando $9,8 \%$ infectados pelo T. cruzi. Na caracterização isoenzimática de 13 amostras de T. cruzi isoladas desses triatomíneos, seis pertenciam ao zimodema $\mathrm{Z} 1$ (ciclo de transmissão silvestre), e sete ao Z2 (ciclo de transmissão doméstico). A captura de exemplares de $P$. megistus no intradomicílio, naturalmente infectados com parasitas de ambos os ciclos, indica a superposição dos ciclos de transmissão da doença de Chagas no município de Bambuí. Outra evidência da inter-relação dos ciclos pôde ser observada no isolamento de T. cruzi Z2 de um gato e a participação do cão como reservatório de $T$. cruzi Z1. A presença do $P$. megistus no peridomicílio representa importante elo entre o ambiente silvestre e o intradomicílio, servindo como veiculador do T. cruzi $\mathrm{Z} 1$ e na manutenção de ciclos de transmissão do T. cruzi $\mathrm{Z} 2$ no peridomicílio e domicílio, propiciando de forma gradual a reinfestação do município caso a Vigilância Epidemiológica seja interrompida.

Palavras-Chave: Doença de Chagas; Trypanosoma cruzi; Ciclos de Transmissão; Reservatórios; Zimodema

\section{REFERÊNCIAS BIBLIOGRÁFICAS}

BAHIA, M. T., 1985. Comportamento Biológico de Cepas de Trypanosoma cruzi no Invertebrado. Tese de Mestrado, Belo Horizonte: Universidade Federal de Minas Gerais.

BARRETTO, M. P., 1967. Estudos sobre reservatórios e vetores naturais do Trypanosoma cruzi. XVII. Contribuição para o estudo dos focos naturais da Tripanosomose Americana, com especial referência à região nordeste do Estado de São Paulo, Brasil. Revista da Sociedade Brasileira de Medicina Tropical, 1: 23-35. , 1979. Epidemiologia. In: Trypanosoma cruzi e Doença de Chagas (Z. Brener \& Z. Andrade, eds.), pp. 89-151, Rio de Janeiro: Guanabara Koogan.

BRONFEN, E.; ROCHA, F. S. A.; MACHADO, G. B. N.; PERILLO, M. M.; ROMANHA, A. J. \& CHIARI, E., 1989. Isolamento de amostras do Trypanosoma cruzi por xenodiagnóstico e hemocultura de pacientes na fase crônica da doença de Chagas. Memórias do Instituto Oswaldo Cruz, 84: 237-240.

CAMARGO, E. P., 1964. Growth and differentiation in Trypanosoma cruzi. I. Origin of metacyclic trypanosomes in liquid media. Revista do Instituto de Medicina Tropical de São Paulo, 6: 93-100.

CARNEIRO, M.; CHIARI, E.; GONÇALVES, A. M.; SILVA PEREIRA, A. A.; MOREL, C. M. \& ROMANHA, A. J., 1990. Changes in the isoenzyme and kinetoplast DNA patterns of Trypanosoma cruzi strains induced by maintenance in mice. Acta Tropica, 47: 35-45.

DEANE, M. P. \& JANSEN, A. M., 1988. From a mono to a digenetic life-cycles: how was the jump for flagellates of the family trypanosomatidae? Memórias do Instituto Oswaldo Cruz, 83: $273-275$. 
DEANE, M. P.; LENZI, H. L. \& JANSEN, A. M., 1984. Trypanosoma cruzi vertebrate and invertebrate cycles in the same mammal host the opossum Didelphis marsupialis. Memórias do Instituto Oswaldo Cruz, 79: 513-515.

DIAS, J. C. P., 1965. Reinfestação do Município de Bambuí por triatomíneos transmissores da doença de Chagas. Memórias do Instituto Oswaldo Cruz, 63: 107-119.

1982. Doença de Chagas em Bambuí, Minas Gerais, Brasil. Estudo Clínico-Epidemiológico a partir da Fase Aguda, entre 1940 e 1982. Tese de Doutorado, Belo Horizonte: Universidade Federal de Minas Gerais. , 1987. Epidemiologia e controle da doença de Chagas no Brasil. Panorama atual. Revista da Sociedade Brasileira de Medicina Tropical, 20 (supl.): 155.

DIAS, J. C. P. \& GARCIA, A. L. R., 1978. Vigilancia epidemiologica con participacion comunitária. Un programa de enfermedad de Chagas. Boletin da Oficina Sanitaria Panamericana, 84: 533-544.

FERNANDES, A. J.; DIOTAIUTI, L.; DIAS, J. C. P.; ROMANHA, A. J. \& CHIARI, E., 1989. Infecção natural das glândulas anais de gambás (Didelphis albiventris) pelo Trypanosoma cruzi no município de Bambuí, Minas Gerais. Memórias do Instituto Oswaldo Cruz, 84: 87-93.

FERNANDES, A. J.; CHIARI, E.; RODRIGUES, R. R.; DIAS, J. C. P. \& ROMANHA, A. J., 1991. The importance of the opossum (Didelphis albiventris) as a reservoir for Trypanosoma cruzi in Bambuí, Minas Gerais State. Memórias do Instituto Oswaldo Cruz, 86: 81-85.

FERNANDES, A. J.; CHIARI, E.; CASANOVA, C.; DIAS, J. C. P. \& ROMANHA, A. J., 1992a. The threat of reintroduction of natural transmission of Chagas' disease in Bambuí, Minas Gerais State, Brazil, due to Panstrongylus megistus. Memórias do Instituto Oswaldo Cruz, 87: 285-289.

FERNANDES, A. J.; ROMANHA, A. J.; STEINDEL, M.; GUIMARÃES, E. C.; DUARTE, A. P. M. \& CHIARI, E., 1992b. Isoenzymaatic characterization of Trypanosoma cruzi clones proceeding from sylvatic and domestic transmission cycles of Chagas' disease in the county of Bambuí, MG, Brazil. Memórias do Instituto Oswaldo Cruz, 87 (supl. II): 100.

FERNANDES, A. J.; DIOTAIUTI, L.; ROMANHA, A. J.; VITOR, R. W. A. \& CHIARI, E., 1993. Participação de reservatórios domésticos na manutenção de focos isolados de transmissão do Trypanosoma cruzi em área de Vigilância Epidemiológica para o controle da doença de Chagas
(Bambuí, MG). Revista da Sociedade Brasileira de Medicina Tropical, 26 (supl. II): 57-58.

FORATTINI, O., 1980. Biogeografia, origem e distribuição da domiciliação de triatomíneos no Brasil. Revista de Saúde Pública de São Paulo, 14: 265-299.

GIBSON, W. C.; MARSHALL, T. F. C. \& GODFREY, D. G., 1980. Numerical analysis of enzyme polymorphism: a new approach to the epidemiology and taxonomy of trypanosomes of the subgenes Trypanosoon. Advance Parasitology, 18: 175-246.

GIBSON, W. C. \& MILES, M. A., 1985. Application of new technologies to epidemiology. British Medical Bulletin, 41: 115-121.

HIDE, G. \& TAIT, A., 1991. The molecular epidemiology of parasites. Experientia, 47: 128-141.

LARANJA, F. S., 1949. Evolução dos conhecimentos sobre a cardiopatia da doença de Chagas. Revisão crítica da literatura. Memórias do Instituto Oswaldo Cruz, 47: 605.

MACÊDO, A. M.; MARTINS, M. S.; CHIARI, E. \& PENA, S. D. J., 1992. DNA fingerprinting of Trypanosoma cruzi: a new toll for characterization of strains and clones. Molecular Biochemical Parasytology, 55: 147-154.

MARTINS, A. V.; VERSIANI, V. \& TUPYNAMBÁ, A. A., 1939/1940. Sobre 25 casos agudos da moléstia de Chagas observados em Minas Gerais. Memórias do Instituto Oswaldo Cruz, 3-4: 01-06.

MILES, M. A., 1983. The epidemiology of South American Trypanosomisis - biochemical and immunological approache and their relevance to control. Transactions Royal Society Tropical Medicine and Hygiene, 77: 05-23.

MILES, M. A.; TOYÉ, P. J.; OSWALD, S. C. \& GODFREY, D. G., 1977. The identification by isoenzyme patterns of two distinct strains groups of Trypanosoma cruzi, circulating independently in a rural area of Brazil. Transaction Royal Society Tropical Medicine and Hygiene, 71: 217225.

MILES, M. A.; SOUZA, A.; POVOA, M.; SAHAW, J. J.; LAINSON, R. \& TOYÉ, P. J., 1978. Isoenzymic heterogeneity of Trypanosoma cruzi in the first autochthonus patients with Chagas' disease in Amazonian Brazil. Nature, 272: 819-821.

OMS (Organización Mundial de la Salud), 1991. Control de la Enfermadad de Chagas. Ginebra: OMS. (Serie de Informes Técnicos, 811)

PELLEGRINO, J. A., 1953. A doença de Chagas em Minas Gerais. Esboço crítico dos trabalhos publicados até 1951. Memórias Instituto Oswaldo Cruz, 51: 611-668. 
READY, P. D. \& MILES, M. A., 1980. Delimitation of Trypanosoma cruzi zymodemes by numerical taxonomy. Transaction Royal Society Tropical Medicine and Hygiene, 74: 238 -242.

ROMANHA, A. J., 1982. Heterogeneidade isoenzimática em Trypanosoma cruzi. Tese de Doutorado, Belo Horizonte: Universidade Federal de Minas Gerais.

SIQUEIRA, A. F., 1960. Estudos sobre a reação de precipitina aplicada à identificação de sangue ingerido por triatomíneos. Revista do Instituto de Medicina Tropical São Paulo, 2: 41-53.

TIBAYRENC, M.; ECHALAR, L.; DUJARDIN, J. P.; POCH, O. \& DESJEUX, P., 1984. The microdistribution of isoenzymic strains of Trypanosoma cruzi in southern Bolivia; new isoenzyme profiles and further arguments against mendelian sexuality. Transaction Royal Society Tropical Medicine and Hygiene, 78: 519-525.

TIBAYRENC, M.; HOFFMANN, A.; POCH, O.; ECHALAR, L.; Le PONT, F.; LEMESRE, L.; DESJEUX, P. \& AYALA, F. J., 1986. Additional data on Trypanosoma cruzi isoenzymic strans encountered in Bolivian domestic transmission cycles. Transaction Royal Society Tropical Medicine and Hygiene, 80: 442.
TIBAYRENC, M. \& AYALA, F. J., 1988. Isoenzyme variability in Trypanosoma cruzi the agente of Chagas' disease: genetical, taxo- nomical and epidemiological significance. Evolution, 42: 277-292.

WHO (World Health Organization), 1978. Proposals for the nomenclature of salivarian trypanosomes and for the maintenance of reference collections. Bulletin World Health Organization, 56: 467480.

ZELEDÓN, R., 1974. Epidemiology, modes of trasmission and reservoir hoste of Chagas' disease. Tripanosomiases and Leishmaniasis with special reference to Chagas' disease. Ciba Foundation Symposium, 20: 51-85. 